\title{
ON DOUBLY INFINITE SYSTEMS OF DIRECTLY SIMILAR CONVEX ARCHES WITH COMMON BASE LINE.
}

BY PROFESSOR E. H. MOORE.

IN his paper "The determination of the constants in the problem of the brachistochrone" (BuLletis, January, 1904, pages 185-188) Professor Bolza has proved analytically the statement of Weierstrass (lectures of 1882) that, of all cycloid arches having bases in a given line and lying on one side of that line, precisely one passes through any two points, lying on that side of the line or one or both lying on the line, and not lying in the same perpendicular to the line.

The special case in which one of the points is on the base line was handled geometrically by the brothers Bernoulli (cf. Ostwald's "Klassiker der exacten Wissenschaften," number 46 , pages 12 and 18), use being made of the fact that all cycloid arches are similar. In reading his paper before the mathematical club of the University of Chicago, November 20, 1903, Professor Bolza, after indicating this solution, added a geometric solution for the case in which the segment is parallel to the base line, use being made furthermore of the fact that a cycloid arch is symmetric with respect to the perpendicular bisector of its base.

In the present note I give an analytically phrased geometric proof of the statement of Weierstrass in its generality, at the same time extending it (cf. theorem $\mathrm{I}_{0}, \S 3$ ) to cover the case of any doubly infinite system of directly * similar convex arches possessing tangents and meeting perpendicularly a common base line.

One obtains an obvious generalization of this theorem in replacing perpendicularity to the base line by parallelism to any given line not parallel to the base line. More essential generalizations remain for consideration. In a subsequent note I propose to take up the connections of this theorem with the calculus of variations.

\footnotetext{
* The distinction between direct and inverse similarity enters only in case the arches do not have the symmetry spoken of above.
} 


\section{$\S 1$. The $\alpha \beta$-Arches.}

With respect to a rectangular $x y$ coördinate system, a curvearc $A B$ joining two points $A=(a, 0), B=(b, 0)$, where $a<b$, is called an $\alpha \beta$-arch, in case its equation

$$
y=f(x)
$$

satisfies the following conditions $1^{\circ}-4^{\circ}$.

$1^{\circ}$.

$$
a \leqq x \leqq b .
$$

$2^{\circ}$. For every $x, a \leqq x \leqq b, f(x)$ is a single valued continuous function of $x$ with a definite (finite or infinite) derivative * $f^{\prime}(x)$.

$$
\begin{aligned}
& 3^{\circ} \text {. If } f^{\prime}\left(x_{1}\right)=f^{\prime}\left(x_{2}\right), a \leqq x_{1} \leqq b, a \leqq x_{2} \leqq b, \\
& 4^{\circ} . \quad f^{\prime}(a)=\alpha \geqq 0, \quad f^{\prime}(b)=\beta .
\end{aligned}
$$

The theorem of the mean is applicable to every arc of an $\alpha \beta$-arch. An $\alpha \beta$-arch $A B$ is readily proved to have the following properties $5^{\circ}-11^{\circ}$.

$5^{\circ}$. A straight line having two distinct points in common with the arch is tangent to the arch at neither of the points and has no third point in common with the arch.

$6^{\circ}$.

$$
+\infty \geqq \alpha>0>\beta \geqq-\infty .
$$

$7^{\circ}$.

$$
f(x)>0 \text { for } a<x<b .
$$

$8^{\circ}$. The transformation

$$
R: \quad \bar{x}=-x, \quad \bar{y}=y,
$$

changes the $\alpha \beta$-arch $A B$ into an $\bar{\alpha} \bar{\beta}$-arch $\bar{B} \bar{A}$, where

$$
\bar{\alpha}=-\beta, \quad \bar{\beta}=-\alpha .
$$

$9^{\circ}$. The transformation

$$
T_{\kappa}: \quad \bar{x}=x, \quad \bar{y}=y-\kappa(x-\alpha) \quad(\alpha>\kappa \geqq 0),
$$

changes the arc $A C$ of the $\alpha \beta$-arch $A B$ into an $\bar{\alpha} \bar{\beta}$-arch $\bar{A} \bar{C}$, with

$$
\bar{\alpha}=\alpha-\kappa, \quad \bar{\beta}=\gamma-\kappa,
$$

* The understanding is that the derivatives $f^{\prime}(a), f^{\prime}(b)$ are respectively progressive, regressive, and that the definite infinite derivatives $+\infty,-\infty$ count as distinct. 
where $C$ is the definite point $(c, f(c)), a<c \leqq b$, common to the arch $A B$ and the line $y=\kappa(x-a)$, and where $\gamma=f^{\prime}(c)$.

$10^{\circ}$. The $\operatorname{arch} A B$ has a single maximum point $M=(m, f(m))$, $f(m)>0, f^{\prime}(m)=0, a<m<b$. A line $y=\eta, 0 \leqq \eta \leqq f(m)$, meets the arch precisely once on each arc $A M, M B$, say at $P_{0 \eta}=\left(x_{0 \eta}, \eta\right), P_{1 \eta}=\left(x_{1 \eta}, \eta\right)$, so that $a \leqq x_{0 \eta} \leqq m \leqq x_{1 \eta} \leqq b$. It is tangent to the arch only in case $\eta=f(m)$. On the arc $A M$, $y$ is a continuous monotonic increasing function of $x$, since otherwise the arch would have two tangents parallel to the $x$-axis, and hence $x$ is such a function of $y$. On the arc $M B, x$ and $y$ are continuous monotonic decreasing functions each of the other. Hence on the interval $0 \leqq \eta \leqq f(m)$, the continuous monotonic function $x_{1 \eta}-x_{0 \eta}$ decreases from $b-a$ to 0 , while the quotient $\eta /\left(x_{1 \eta}-x_{0_{\eta}}\right)$, continuous except for $\eta=f(m)$ and monotonic, increases from 0 to $+\infty$, and hence assumes precisely once any particular finite value $\lambda \geqq 0$.

$11^{\circ}$. On the interval $a \leqq x \leqq b f^{\prime}(x)$ is a continuous monotonic function decreasing from $\alpha$ to $\beta$.- This follows readily from $8^{\circ}, 9^{\circ}, 10^{\circ}$.

\section{$\S 2$. The Group $G_{2}$ and the $\kappa \lambda$-Segments.}

The transformations

$$
T_{k, l}: \quad \bar{x}=k x+l, \quad \bar{y}=k y
$$

constitute a two parameter group, $G_{2}$, generated by the translations $T_{1, l}$ and the expansions $T_{k, 0^{\circ}}$

Under a transformation $T_{k, l}^{1,0^{*}}$ an $\alpha \beta$-arch changes into an $\alpha \beta$-arch, identical with the former only if $T_{k, l}$ is the identity $T_{1,0^{*}}$ Hence under the group $G_{2}$ an $\alpha \beta$-arch changes into $\infty^{2}$ $\alpha \beta$-arches constituting a two-parameter system of such arches equivalent under the group.

A pair of points

$$
P_{0}=\left(x_{0}, y_{0}\right), \quad P_{1}=\left(x_{1}, y_{1}\right) \quad\left(x_{0}<x_{1}, y_{0} \geqq 0, y_{1} \geqq 0\right)
$$

determines a so-called $\kappa \lambda$-segment $P_{0} P_{1}$, where

$$
y_{1}-y_{0}=\kappa\left(x_{1}-x_{0}\right), \quad y_{1}+y_{0}=2 \lambda\left(x_{1}-x_{0}\right) .
$$

The constants $\kappa, \lambda$ are characteristic invariants of the segment $P_{0} P_{1}$ under the group $G_{2}$, that is, if $P_{0} P_{1}$ and $\bar{P}_{0} \bar{P}_{1}$ are equivalent under $G_{2}$, then $\kappa=\kappa, \lambda=\bar{\lambda}$, and, conversely, if 
$\kappa=\bar{\kappa}, \lambda=\bar{\lambda}$ then $P_{0} P_{1}$ and $\bar{P}_{0} \bar{P}_{1}$ are equivalent under $G_{2}$, indeed by means of a single transformation $T_{k, l}$ of $G_{2}$.

These characteristic invariants $\kappa, \lambda$ are subject merely to the conditions $\kappa=$ any, $\quad \lambda \geqq 0$.

$\S 3$. The Circumscription and Inscription Theorems.

Theorem I. Precisely one of a given system of $\infty^{2}$ a $\beta$-arches equivalent under the group $G_{2}$ circumscribes a given $\kappa \lambda$-segment, where $\alpha>\kappa>\beta$.

An immediately evident corollary of theorem $I$ is

Theorem $\mathrm{I}_{0}$. Precisely one of a given system of $\infty^{2}$ $(+\infty,-\infty)$-arches equivalent under the group $G_{2}$ circumscribes a given $\kappa \lambda$-segment.

Such a system is, for instance, the system of semicircular arches with basal diameters on the $x$ axis, and another is the system of cycloid arches with bases on the $x$ axis.

In view of the remarks of $\S 2$, theorem $I$ is equivalent to

Theorem II. Precisely one $\kappa \lambda$-segment $(\kappa, \lambda$ given $)$ is inscribed in a given $\alpha \beta$-arch, where $\alpha>\kappa>\beta$.

Since (by $\$ 1,6^{\circ}$ )

$$
+\infty \geqq \alpha>0>\beta \geqq-\infty,
$$

the proof of theorem II naturally falls into three parts:

The case $\kappa=0$ is proved in $\S 1,10^{\circ}$.

The case $\alpha>\kappa>0$ is reduced to the preceding case $\kappa=0$, by the transformation $T_{\kappa}$ of $\S 1,9^{\circ}$. For (in the notations there used) the arcs $A C, C B$ lie on opposite sides of the line $A C$, so that any $\kappa \lambda$-segment inscribed in the arch $A B$, being parallel to the line $A C: y=\kappa(x-a)$, must be inscribed in the arc $A C$ or in the arc $C B$. There is none in the arc $C B$, since in that case each arc $A C, C B$ would have a tangent parallel to the line $A C$. In the arc $A C$ however there is precisely one; for any $\kappa \lambda$-segment $P_{0} P_{1}$ inscribed in the arc $A C$ transforms by $T_{\kappa}$ into a $\bar{\kappa} \bar{\lambda}$-segment $\bar{P}_{0} \bar{P}_{1}$ inscribed in the $\bar{a} \bar{\beta}$ $\operatorname{arch} \bar{A} \bar{C}$, where

$$
\begin{gathered}
\bar{\alpha}=\alpha-\kappa, \quad \bar{\beta}=\gamma-\kappa, \\
\bar{\kappa}=0,2 \bar{\lambda}=2 \lambda-\kappa \frac{x_{1}+x_{0}-2 \alpha}{x_{1}-x_{0}}=\frac{\bar{y}_{1}+\bar{y}_{0}}{\bar{x}_{1}-\bar{x}_{0}}
\end{gathered}
$$

so that $\bar{\lambda} \geqq 0$, since $\bar{y}_{0} \geqq 0, \bar{y}_{1} \geqq 0,-$ and conversely; and by 
the preceding case there is precisely one such $\bar{\kappa} \bar{\lambda}$-segment $\overline{P_{0}} \overline{P_{1}}$ inscribed in the $\bar{\alpha} \bar{\beta}$-arch $\bar{A} \bar{C}$.

The case $0>\kappa>\beta$ is reduced to the preceding case $\alpha>\kappa>0$, by the transformation $R$ of $\S 1,8^{\circ}$. For under $R$ a $\kappa \lambda$-segment $P_{0} P_{1}$ transforms into a $\kappa \bar{\lambda}$-segment $\overline{P_{1}} \bar{P}_{0}$, and an $\alpha \beta$-arch $A B$ transforms into an $\bar{\alpha} \bar{\beta}$-arch $\bar{B} \bar{A}$, where

$$
\bar{\kappa}=-\kappa, \quad \bar{\lambda}=\lambda ; \bar{\alpha}=-\beta, \quad \bar{\beta}=-\alpha,
$$

so that indeed $\bar{\alpha}>\bar{\kappa}>0$.

The University of Chicago,

February 6, 1904.

\section{THE RICCATI DIFFERENTIAL EQUATIONS \\ WHICH REPRESENT ISOTHERMAL SYSTEMS.}

BY DR. EDWARD KASNER.

( Read before the American Mathematical Society, December 29, 1903.)

THE object of this note is to determine the isothermal systems of (plane) curves which can be represented by an equation of Riccati type

$$
y^{\prime}=P+Q y+R y^{2}
$$

where $P, Q, R$ denote arbitrary functions of $x$. The result obtained is that there are four distinct systems of this kind, namely, those given by the equations (3), (4), (6), (7) below.

It has been shown by Lie* that the differential equation of any isothermal system is of the form

$$
y^{\prime}=\tan F(x, y)
$$

where $F$ is a harmonic function; that is,

$$
F_{x x}+F_{y y}=0 \text {. }
$$

By a simple transformation this result may be expressed more conveniently for the present purpose as follows :

* Lie-Scheffers, Differentialgleichungen, 1891, p. 157. 